\title{
LA EDUCACIÓN DEL ESPÍRITU. LÍNEAS DE PENSAMIENTO PARA UNA TEORÍA DE LA EDUCACIÓN
}

\author{
THE EDUCATION OF THE SPIRIT. \\ LINES OF THOUGHT FOR A THEORY OF EDUCATION
}

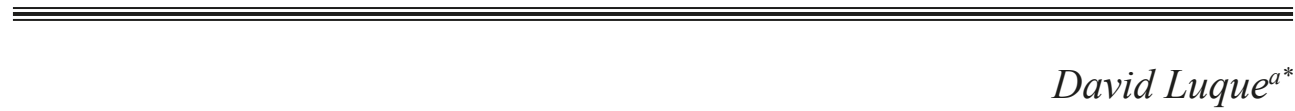

Fechas de recepción y aceptación: 27 de mayo de 2019, 11 de octubre de 2019

DOI: 10.46583/edetania_2020.57.453

Resumen: Introducción. Este artículo se sitúa en la rica y profunda discusión internacional sobre la educación del espíritu que contrasta con un silencio espeso sobre el tema en el ámbito pedagógico. Método. Articula los pasos clásicos de una teoría de la educación. Ofrece una aproximación filosófico-educativa al "espíritu" para terminar preguntándose por sus aplicaciones prácticas. Discusión. Busca identificar unos rasgos dinámicos que permitan intervenir educativamente en el espíritu, saber si es legítimo epistemológicamente y cómo se relaciona internamente con otras áreas de la educación. Conclusión. Comprendido el espíritu de una cierta manera, parece posible atender a su educación en contextos religiosos y seculares.

Palabras clave: desarrollo del espíritu, pensamiento crítico, valores morales, educación pública.

Abstract: Introduction. This article is set in the rich and deep international discussion on the education of the spirit that contrasts with an absolute silence on the subject in the pedagogical field. Method. It articulates the classic steps of a theory of education. It offers a philosophical-educational approach to the "spirit" to end up wondering about its practical

${ }^{\text {a }}$ Facultad de Ciencias Sociales y Jurídicas. Universidad Rey Juan Carlos.

${ }^{*}$ Correspondencia: Universidad Rey Juan Carlos. Facultad de Ciencias Sociales y Jurídicas. Camino del Molino, s/n. 28943 (Fuenlabrada), Madrid. España.

E-mail: david.luque@urjc.es 
applications. Discussion. It seeks to identify dynamic traits that allow educational intervention in the spirit, to know if it is legitimately epistemologically and how it is internally related to other areas of education. Conclusion. Understanding the spirit in a certain way it seems possible to attend to their education in religious and secular contexts.

Keywords: Spiritual Development, critical thinking, moral values, public education.

\section{INTRODUCCIÓN O ENTRE EL SILENCIO Y LA DISCUSIÓN}

"Si no cierras bien los ojos, muchas cosas no se ven"

Fito \& FITIPALDIS

Este artículo, cuyo fin es estudiar la educación del espíritu ${ }^{1}$, se sitúa entre el silencio y la discusión.

El silencio se refiere al hecho de que gran parte de la comunidad científica rechaza su estudio. Quienes lo refutan aducen la escasa claridad conceptual del "espíritu", que no es posible describirlo con la precisión metodológica con que se analiza el intelecto, el cuerpo o incluso lo moral (Beringer, 2000, pp. 157-162). Además, la contraposición entre racional e irracional desecha la comprensión mistérica del fenómeno (Filipsone, 2009, p. 122). Casi como atributo añadido se encuentran aquellas razones derivadas de la identificación entre "espíritu" y "judeocristianismo", lo que provoca que allí donde se rechaza lo religioso en la educación se rechace, por ende, las posibilidades de educar el espíritu en espacios públicos (Dayton, 2015, pp. 432-433). Justo aquí se encontraría la última razón: los problemas derivados de articular lo que sea que termine siendo una ES en el tejido democrático actual.

Por su parte, la discusión, que ha sido ingente y profunda en el contexto internacional, se ha visto sostenida y alentada por otros tantos factores. El primero, la presencia constante del espíritu en pedagogos como Rudolf Steiner (Goldshmidt, 2017, pp. 4-14), John Dewey (Webster, 2009, pp. 96-102) o Paulo Freire (Kirylo, 2017, pp. 529-594). Además, la necesidad de una pedagogía integral lleva a considerar el espíritu como una dimensión más de la estructura humana que debe ser educada (Crossman, 2003, pp. 505-508). En

${ }^{1} \mathrm{ES}$, en adelante. 
tercer lugar, se podría trazar un cierto paralelismo entre los argumentos para defender una educación moral en el ámbito público y los argumentos para hablar de una ES (Dayton, 2015, pp. 432-435). Esta discusión se ha visto alentada por la exigencia de una ES en las leyes educativas promulgadas por el Gobierno británico (Wright, 2000, pp. 61-69). Todo ello demanda, en definitiva, una gran apertura de mente y una gran creatividad a la hora de hacer filosofía de la educación (Hansen, 2019).

Pues en esa tensión entre el silencio y la discusión nace este artículo, que articulará una estructura en tres partes. El primer apartado analiza si es posible hablar de un desarrollo del espíritu que permita sostener los postulados básicos de una intervención pedagógica. El segundo intenta realizar una aproximación a la discusión sobre la posibilidad epistemológica de una ES, toda vez que parece existir una dinámica interna. Y el tercero discute las aportaciones de la ES a algunas coordenadas fundamentales de la educación desde un punto de vista intrarreligioso y secular.

Comencemos.

\section{El desarrollo del espíritu. Fundamento para una intervención PEDAGÓGICA}

Como acabo de señalar, aunque un problema genético en la ES es la ausencia de un concepto que delimite todos los atributos del mismo espíritu, se entiende que acaso sea un componente dinámico en la estructura antropológica del ser humano que es susceptible de ser educado (Adams, Bull y Maynes, 2015, p. 4; Benson et al., 2012, p. 454; Benson, Roehlkepartain y Rude, 2003, p. 205). Pero aquí parece más importante el análisis de su desarrollo que la propia definición, puesto que allí donde hay crecimiento hay posibilidades de consideración educativa. Para hacerlo se compondrán dos epígrafes que analizarán los datos alcanzados en el campo de estudio y las aportaciones desde el punto de vista intrarreligioso. 


\subsection{Los hallazgos en el campo del desarrollo del espíritu}

Con todo, hay una serie de evidencias que sirven para enmarcar este análisis, especialmente en lo que se refiere a la infancia. Para los niños, Dios tiene una realidad objetiva casi incuestionable (Wills, 2012, p. 54) y se da una fuerte espiritualidad que se diluye progresivamente (Hay, 2000, pp. 40-43) a la vez que se vuelve más personal en quienes la mantienen cuando se encuentran ya en la adolescencia (Mata-McMahon, 2016, p. 4). En cualquier caso, incluso desgajada de un contexto religioso, es unánime que la ES parece un terreno fértil para la educación, la madurez y la felicidad (Benson, Roehlkepartain y Rude, 2003, pp. 205-207).

Dicho lo cual, ¿qué hemos comprendido de su desarrollo? A la vista de las investigaciones realizadas, parecen existir cinco rasgos comunes en los que se sustenta la espiritualidad en la infancia. Todo señala que la "notion of relationality is at the core" (Adams, Bull y Maynes, 2015, p. 4). En efecto, las relaciones constituyen el elemento central de la espiritualidad en la infancia y sintetizan el mundo del niño, a saber, "relationships with self, other, the world and God/the trascendent" (Adams, Bull y Maynes, 2015, p. 4). De aquí nacería la necesidad de conocerse a sí mismos. Este conocimiento crece en la búsqueda de significado materializada en las preguntas infantiles sobre las cuestiones últimas de la realidad (Adams, Bull y Maynes, 2015, pp. 4-5; Duff, 2003, p. 232) y la propia narrativa del niño que intenta hacer inteligible su vida a los demás -volveré a ello más tarde-. Aquí, de nuevo, el componente relacional adquiere una dimensión espiritual. Porque la relación que los niños mantienen con otros seres humanos excede los límites de la mera socialización. "Connectedness refers to a deeper level than that of having friends and feeling part of community [...]. The very sense of being connected to others (whether to other people, creatures or things) is intangible, it goes beyond anything that is visible or tactile; it is, in essence, spiritual" (Adams, Bull y Maynes, 2015, p. 5; Jones, 2005, pp. 4-5). En la literatura científica se subraya con insistencia la idea del momento presente como el espacio donde nace lo espiritual. "The 'everyday' is often used to indicate how children's spirituality is expressed in ordinary events can easily be overlooked by adults" (Adams, Bull y Maynes, 2015, p. 9; ver también Wills, 2012, pp. 5759). Schinkel atribuyó esta capacidad al concepto wonder, a la capacidad de 
maravillarse. Dice: "in the case of deep wonder, the «strange» takes on the form of deeply other or mysterious, which present itself not just as beyond our present ability to understand it, but as fundamentally beyond the limits of our understanding" (Schinkel, 2018, p. 34). La capacidad de trascender, por su parte, irrumpe en dos sentidos. Por esa búsqueda de significado constante y por la capacidad de ir más allá del momento presente precisamente en la búsqueda de ese propósito (Adams, Bull y Maynes, 2015, pp. 4-5; Jones, 2005, pp. 3-4).

La adolescencia y la entrada en la madurez son ámbitos del desarrollo espiritual menos estudiados en la literatura científica (Kilcup, 2015, p. 2; King y Boyatzin, 2004, pp. 2-5; Zajonc, 2003, pp. 50-52). Más que una dinámica, Kilcup insiste en la idea de describir rasgos. Que muchos adolescentes tienen "spiritual experiences, holding spiritual values, and conducting deep existential searching" (Kilcup, 2015, p. 13). Y muchos adolescentes que se declaran ateos o agnósticos viven fuertes experiencias espirituales. En general, la espiritualidad adolescente reside, también, en una búsqueda de sentido que, en fin, puede darse al margen de una vida ascética. "Thus", concluye Kilcup, "spiritual experiences might happen organically for some adolescent individuals" (Kilcup, 2015, p. 13).

Muchos de los rasgos que acaban de aparecer imponen su presencia, también, en un contexto intrarreligioso.

\subsection{El desarrollo del espíritu dentro de las grandes religiones}

En efecto, y aunque no trataré específicamente cada una de las religiones, ellas añaden elementos que matizan y enriquecen lo conocido hasta aquí.

En gran parte, las religiones orientales subrayan lo señalado anteriormente: la importancia de la consciencia del momento presente, el autoconocimiento y la trascendencia unitiva (Duff, 2003; McCann, 2009, pp. 918-926). Por su parte, las religiones del Libro amplían los datos. En el islamismo, donde la "primary obligation of the human being is to listen and obey", la ES "emphasises the behavioural or Code aspect of religion" (McCann, 2009, p. 933). A su manera, el judaísmo aparece también vinculado a la Tôrâ (McCann, 2009, pp. 927-930) que conduce la ES al dominio de un corpus filológico refinado 
que permita acceder a la Escritura, donde hay una presencia real de Yahvé, y a una apertura ética al prójimo, por mandato bíblico y en espera del mesías (Zweiback, 2015). Aunque el cristianismo también está unido indefectiblemente a la Biblia, su dependencia textual no es tan marcada desde el punto de vista de la ES (Ruíz Salvador, 1998, pp. 379-718). Aparece vinculada a la filiación divina por medio de Jesucristo y en el Espíritu. Y, aún dentro de estas vinculaciones, en todas las religiones existe una amplia variedad de carismas que amplía las formas de vida espirituales. Con todo, la literatura científica ha comprendido algunos elementos esenciales desde el punto de vista de la religión en su relación con la espiritualidad.

La adquisición de un lenguaje religioso desempeña un papel fundamental en la ES. Aquí la comunidad científica aparece dividida entre quienes piensan que el individuo escoge una religión porque esta posee un lenguaje que da acomodo lingüístico a su experiencia o que es el lenguaje específico el que da forma a la experiencia (Radford, 1999, pp. 168-170; Benson et al., 2012, 455; Reimer y Furrow, 2001, pp. 7-10). Pero, con independencia de esta división, se observa cierta unanimidad en que la importancia de usar el lenguaje para expresar la experiencia espiritual a otros seres humanos (Roebben, 2014, pp. 306-307; Reimer y Furrow, 2001). Esta necesidad de comunicar las experiencias tiene su importancia en varios sentidos: incide en que el fundamento del desarrollo del espíritu tiene una dimensión comunitaria -ya hay estudios que demuestran la importancia tanto de comunicar los encuentros con lo divino (Lovelock y Adams, 2017, pp. 9-11) como de ser acompañado en la vida espiritual (Marina Díaz, 2016, pp. 6-10)-; ofrece una clave de interpretación de la propia existencia a la luz de las experiencias espirituales, que se revelan como una clave hermenéutica del sentido de esta al focalizar la atención sobre la relación del individuo en comunidad y con la trascendencia (MataMcMahon, 2016, pp. 7-9; Radford, 2007; Reimer y Furrow, 2001, p. 16); la interpretación de la propia vida en la comunicación con los demás dota de sentido la propia existencia al percibir una unidad sostenida por lo trascendente (Roebben, 2014, 305-306; Geiger, 2015, pp. 174-176; Watson, 2011, pp. 99-100). Con todo, es necesario señalar unas salvedades a estos rasgos. La educación, incluso la ES en contextos públicos, parece desempeñar un papel muy secundario en los procesos tanto de elección de una religión como 
de conversión (Benson, 2012, p. 455). Además, a pesar de que la ambición del estudio del desarrollo del espíritu en términos seculares y religiosos es marcar un itinerario formativo, el componente fundamental está vinculado a la propia persona (Benson, 2003) -lo que significa que es cuestionable una espiritualidad que tiende a la uniformización.

$* * *$

En definitiva, ¿qué desarrolla la ES desde el punto de vista de su dinámica tanto en el ámbito de estudio, la disciplina, como en su relación con las religiones? Aunque serían necesarios muchos matices, Benson y sus compañeros anotan las siguientes dimensiones: "becoming aware of one's potential and possibility; becoming aware of the intersection of one's life with others, nature, and the universe; connecting and linking the self and one's potential to ideals and narratives; and developing a life orientation that generates hope, purpose and compassion" (Benson et al., 2012, p. 457). Pero habiendo discernido estas dinámicas la pregunta que se abre ahora, entonces, tiene que ver con la posibilidad epistemológica de la existencia misma de una ES. Porque a pesar de este desarrollo, ¿puede existir algo así como una ES? Y si existe, ¿cómo lo hace?

3. DisCuSIONES EPISTEMOlÓGICAS SOBRE LA POSIBILIDAD DE UNA EDUCACIÓN DEL ESPÍRITU. UN INTENTO DE CLARIFICACIÓN

La complejidad de la respuesta a las preguntas anteriores no parece concluirse con una definición del concepto espíritu, sino que está íntimamente relacionada con su existencia pedagógica. Así, el desarrollo epistemológico se ha producido en dos núcleos agonísticos cuyos protagonistas son el profesor David Carr, por un lado, y el profesor Hanan A. Alexander, por otro. Son ellos quienes estructuran este apartado. 


\subsection{De David Carr a Michael Hand. La educación del espíritu en la filosofía analítica de la educación}

El pensamiento de Carr se sitúa en los postulados de la filosofía analítica, una comprensión eminentemente aristotélica de la educación y un marco teológico cristiano. Como digo, estas fuentes informan los argumentos que desarrollo a continuación.

En efecto, Carr percibió que "the analytical philosophy of spiritual education is still in a state of relative infancy and there is urgent need for some basic conceptual geography in the field" (Carr, 1995, p. 84). Tal geografía debía dibujar una posible ES para las instituciones públicas -algo nada senci1lo, puesto que él incluso señaló tres formas de entenderla (Carr, 2000)-. Sea como fuere, concluyó que la ES se nutre de ideales y metas que exceden lo mundano y, por lo tanto, se dirige a desarrollar las disposiciones intelectuales y morales necesarias para el logro de esos valores sobrenaturales (Carr, 1995, p. 90). Según esto se pueden distinguir dos dimensiones pedagógicas. Un tipo de conocimiento espiritual común a todos los seres humanos que nace al interpretar determinados pasajes de la Biblia (Carr, 1995, pp. 86-88). Y un tipo de manifestación moral del espíritu en las virtudes cardinales que guardan paralelismos internos con las teologales (Carr, 1995, pp. 91-93). La consecuencia del argumentario es que la ES no puede identificarse con una asignatura, sino que inspira el curriculum de ciertas disposiciones espirituales que se expresarían en el mero hecho de maravillarse ante la vida que se abre en los procesos biológicos, el misterio abisal de las matemáticas o la propia experiencia espiritual que proporciona el goce estético (Carr, 1995, pp. 94-95). Las respuestas a Carr no se hicieron esperar.

La primera la propuso el profesor Blake. Su contrargumentación sostenía que la ES solo puede acontecer en las instituciones seculares como continuación de la fe en que se generan (Blake, 1996, p. 444). Pero la identificación entre espiritualidad y trascendencia cristiana es contingente, es decir, puede acontecer con otro tipo de trascendencia incluso mundana (Blake, 1996, p. 453) y, de cualquier modo, constituye "a minor aspect of our lives, not a major one" (Blake, 1996, p. 452). La respuesta de Carr no aporta una información determinante (Carr, 1996, pp. 457-463), a diferencia de las dos líneas 
de pensamiento que ofrece el profesor Lewis, quien refutará tanto a Carr como a Blake. Que la ES debe comprenderse como un todo integrado en los fenómenos de la realidad, la estructura de la persona y la educación. Y que es el cultivo de las cualidades más profundas de la mente y el corazón (Lewis, 2000). Pero de todas las ideas aparecidas acaso la mayor aportación desde la perspectiva de la filosofía analítica -en mi opinión, mayor incluso que la del propio Carr- la efectúa Michael Hand. Acomete su empresa a partir de aquella idea de bosquejar una geografía que permita moverse en el entramado pedagógico a que da lugar una ES. Según esto deduce cuatro posibilidades epistemológicas. La primera sería una "education based on spiritual principles" (Hand, 2003, p. 397), a saber, una educación que nace de principios religiosos que terminan orientando las dimensiones teoréticas y morales de la educación. La segunda interpretación tendría que ver con la educación del espíritu humano. Sería justamente la que Hand cree que puede establecerse como una educación propia de los contextos seculares, toda vez que rechaza el resto de interpretaciones por su vinculación demasiado estrecha con marcos de pensamiento religiosos. En sus propias palabras, sería "a call for educational attention to the heart as well as the head. It would be an assertion that our responsabilities as educators extend beyond the transmission of knowledge and understanding to the firing of passions and the shaping of character" (Hand, 2003, p. 398). La tercera categoría de la ES sería aquella que educa las prácticas devocionales que permiten al ser humano entrar en comunión con lo divino. Y la última sería la educación de la actitud de esas personas que muestran "certain serenity or equanimity" o "detached from the pressures and anxieties that normally attende very day life" (Hand, 2003, p. 399).

En definitiva, se pueden establecer dos ideas básicas: que resulta muy problemático desvincular una ES de un contexto religioso y que la vía que parecen vislumbrar añade cualidades a la educación del intelecto y a la educación moral que estas, por sí mismas, no alcanzan a cultivar. Hanan A. Alexander acometerá el reto al margen ya de los postulados de la filosofía analítica. 


\subsection{Hanan A. Alexander y la educación del espíritu. Tradición, pensamiento crítico y comunidad}

También Alexander aspira a localizar el fundamento que legitime una ES en la discusión pública. Los ejes que vertebran su pensamiento son el semitismo y que no se circunscribe a la filosofía analítica -acaso su empresa nace de lo ético y se extiende a lo político-.

A Reclaiming goodness lo atraviesan tres líneas de pensamiento fundamentales. Según la primera línea de pensamiento parece existir una identificación entre espiritualidad y ética que mana de dos fuentes: las consecuencias racionalistas de la Ilustración, que han diluido las tradiciones que transmitían un sentido de la existencia, y la formación de una espiritualidad inteligente que se erige como el camino para alcanzar ese "goodness", esa bondad que "can be conceived in terms of four criteria (1) it is an ethical concept; (2) it is holistic; (3) it is pragmatic; and (4) it is synthetic" (Alexander, 2001, p. 42). Para la segunda línea de pensamiento las espiritualidades que buscan esa bondad pueden clasificarse en subjetivas, objetivas o colectivas, sabiendo que es el equilibro interrelacionado de las tres lo que consigue formar una espiritualidad que se despliega en la bondad (Alexander, 2001, pp. 62-141). Este proceso conduciría a una ES que es "acquiring authentic identities in learning communities that teach visions of a higher good" (Alexander, 2001, p. 141). Y para la tercera línea de pensamiento esas "learning communities" son el núcleo donde acontece la ES. Son comunidades vinculadas a tradiciones teológicas que transmiten una interpretación de la realidad para ayudar a adquirir los "higher goods" a través de una racionalidad crítica que discierna ese sentido y despliegue de unas virtudes que conformen un carácter. Por su parte, las críticas del profesor Gary, que son las que llamaron la atención de Alexander, pueden concretarse en tres elementos.

Gary no estaba de acuerdo con esa interpretación ética de la ES. Sostenía que era necesario añadir que los seres humanos son falibles y no pueden crecer espiritualmente debido a causas internas (Gary, 2006, p. 323). Además, la ES que acontece en las learning communities está ligada a tradiciones generalmente teológicas $\mathrm{y}$, en ese sentido, parecen identificarse con sinagogas o campamentos judíos de verano, iglesias protestantes o parroquias católi- 
cas que no responden al desafío de una ES en una democracia (Gary, 2006, p. 324). De esta apreciación se deduce precisamente que, para defender una ES en democracia y para salvar la posible crítica de adoctrinamiento religioso que nacería de la descripción anterior, Alexander subrayó que el pensamiento crítico era imprescindible hasta el punto de constituir "the very center of spirituality" (Gary, 2006, p. 325). Contra esto, Gary distingue entre ratio e intellectus, donde la primera se identifica con la acción y la segunda con la contemplación. En su opinión, la ES viviría en el intellectus. Ante esto, Alexander profundizó en sus argumentos. Puntualizó que entendía la relación entre la espiritualidad y la moralidad como una reflexión sobre la vida buena, no solo sobre las normas que se despliegan en la vida ética (Alexander, 2006, pp. 327-330). Y que el tipo de conocimiento que asocia a la espiritualidad tiene que ver con el concepto de phronesis, esto es, un conocimiento comprendido en términos prácticos y teleológicos -no meramente técnicos (Alexander, 2006, pp. 330-333)-.

De Alexander son sustanciales dos ideas. La necesidad de un pensamiento crítico en la vida espiritual que permite una comprensión crítica de la vida buena que, a la postre, forja un carácter. Y que esa imagen se vive en comunidades de aprendizaje donde se transmite el sentido de la vida buena y las prácticas que conducen a vivirla éticamente.

$$
* * *
$$

Existen otras discusiones epistemológicas menores sobre la ES que hablan de la relacionalidad subyacente tras la unidad de la realidad (Lingley, 2016; De Souza, 2017) y que resignifica principios fundamentales de la educación liberal (Marples, 2006; Best, 2008). Pero, hasta aquí, es posible concluir tres cosas. Que la ES puede existir en contextos intrarreligiosos y seculares, incluso revitalizando las democracias. Que su lugar no se reduce a una asignatura, sino que informa desde dentro la educación intelectual y moral en una relación recíproca. Y que es una experiencia comunitaria. A la postre, esta legitimidad epistemológica abre la posibilidad a discutir una teoría de la educación del espíritu internamente. 


\section{UNA TEORÍA DE LA EDUCACIÓN DEL ESPÍRITU}

Un leve indicio de esta posibilidad acaso resida en que ya existen interpretaciones metafóricas de esta ES (p. e. Caranfa, 2004; Jones, 2005; Ho Wong, 2006; De Souza, 2016). Pero el decurso de la argumentación, ahora, exige otra pregunta: ¿cómo se relaciona la ES con otras dimensiones del proceso educativo? Para responder volveré a centrarme específicamente en análisis que atiendan separadamente las posibilidades intrarreligiosas y seculares.

\subsection{Influjo de principios espirituales en el ámbito intelectivo y moral de la educación desde un punto de vista intrarreligioso}

Desde un punto de vista intrarreligioso, la ES conduciría a una lectura espiritual de algunos elementos de la educación -en la que no me centraré por razones de espacio (Mayes, 2002; Roebben, 2017; Burbules, 2004; Alexander, 2001; De Marzio, 2017; Ben-Peretz, 1975; Geiger, 2015; Roebben, 2014)-. La lectura que reclama más la atención, aquí, es el estudio del componente intelectivo y moral de la educación religiosa a la luz de la ES.

Desde un punto de vista intelectivo, acaso todo se concretaría en las categorías relación y unidad como generadoras de sentido. Ya aparecieron previamente algunas consideraciones gnoseológicas al sugerir que las religiones buscan no tanto educar una mente erudita como una capaz de comprender la realidad desde la presencia de Dios en ella. Pero esa mirada, y aquí radica la novedad de este epígrafe, solo puede ser educada si se refleja en un currículum que reproduzca las relaciones internas de la realidad, la profundidad de conocimientos espirituales objetivos y refiera todo a Dios. Aquí aparecen dos grandes figuras: los beatos Antonio Rosmini y John Henry Newman. Para el teólogo italiano, la unidad constituye el punto focal desde el que nace su pedagogía: hay una unidad de la persona humana, donde lo intelectual se abre a lo moral y lo moral -inspirado en el amor- a lo trascendente, una unidad de la realidad, que está regida por una ley de gradación, y una unidad del método pedagógico, que es el elemento que une persona y realidad en el deber-ser (Rosmini, 2011). El currículum nacería justamente como la destilación de todas esas consideraciones: una gradación progresiva que señale la actuación 
de Dios en la historia humana (Muratore, 1998, pp. 247-261, esp. pp. 249253). Por su parte, el teólogo inglés atribuía a la teología la función unitiva en el currículum, pues, como ciencia que refleja la acción creadora y la presencia continua de Dios, lograba ese mismo efecto al salvaguardar los excesos de las otras ciencias y sintetizar sus aportaciones integrándolas en el discurso teológico (Newman, 2009, pp. 50-51, 78-80 y 96-97). En el contexto de una educación básica, los argumentos resuenan en las propuestas donde los aprendizajes plantean problemas que demandan a los estudiantes no solo un trabajo autónomo y colaborativo a partes iguales, sino encontrar respuestas que proceden desde todas las parcelas de la realidad para articular una única solución al problema o proyecto propuesto. En el ámbito universitario parece asentarse la idea del core curriculum, es decir, un grupo de asignaturas comunes a todas las carreras que ofrecen contenidos que ayudan a dotar de sentido la realidad y los propios estudios. Justo aquí, al final, habría que considerar todavía la capacidad de algunos contenidos para generar preguntas de naturaleza espiritual, es decir, que tienen que ver con el sentido de la propia vida y la realidad, así como que la relación también ha de abrirse necesariamente al conocimiento de otras religiones diferentes a la propia. Pero esto casi abre ya consideraciones de naturaleza moral.

Desde un punto de vista moral, la discusión es más compleja. Ya señalé que Carr y Alexander quisieron integrarla en el entramado de la educación de las virtudes o del carácter, bien atribuyendo paralelismos entre las virtudes cardinales o teologales, bien identificando nuevas virtudes. Pero ellos, como los grandes teólogos de la historia, no consiguieron discernir bien la relación interna entre las virtudes cardinales y aquellas teologales que se identifican como propias del espíritu. Con todo, parece que existen tres presupuestos básicos. Que las virtudes teologales expresan la vida religiosa del hombre de fe en un dinamismo interno que casi exige que hablemos de ellas como de una unidad. Que hay una relación entre la vida moral y la vida religiosa por cuanto que la vida del hombre adquiere una materialización específica en su praxis -aunque la vida moral no agota la vida religiosa-. Y, como ya he dicho, que no conocemos el sistema interno por el que interactúan entre sí. No obstante, parece que el discurso sobre la formación moral desde la perspectiva de la ES en un entramado intrarreligioso conduce a pensar en el amor. Ahí encontramos que tanto las religiones orientales como incluso el mundo 
secular tienen asumida la llamada "ley de oro". Y en el judeocristianismo el discurso no parece muy lejano. El shema, que fija la primacía normativa de amar a YWVH (Dt 6, 4 s), ha de leerse junto a otro de los términos básicos de la Alianza, el mandato que ordena "ama al prójimo como a ti mismo" (Ex 20,1-17; Dt 10,19; Lev 19, 18.32). El cristianismo establece unos lineamientos exegéticos que van desde la comprensión ontológica de Dios en "Dios es amor" (1 Jn 4,8) hasta la orientación práctica en "Os doy un mandamiento nuevo: que os améis los unos a los otros; que, como yo os he amado, así os améis también entre vosotros. Todos conocerán que sois discípulos míos en una cosa: en que os tenéis amor los unos a los otros" (Jn 13,34; también Rm 5, 8; Cor 13, 2; 1 Jn 4,12). El magisterio reciente de la Iglesia parece condensar estas intuiciones en una relectura antropológica del ser humano que refleja la dinámica interna de la vida intratrinitaria (cfr. Juan Pablo II, 1986; Benedicto XVI, 2007, nn. 7-8; 2009, nn. 54-57, esp. 54; Francisco, 2013, n. 178; 2015, n. $240 ; 2016$, n. 324; 2019, nn. 112-133, 163-167). Y así ayer como hoy, cuando son retomadas estas intuiciones por el personalismo semítico (Cohen, 2004) y la ontología trinitaria (Hemmerle, 2005). ¿Pero qué concluir de este breve excursus? Acaso que el amor, que encuentra su expresión transreligiosa en la "regla de oro", constituye el cauce de expresión moral de la vida espiritual y, por lo tanto, el núcleo desde el que ha de pensarse una ES en términos morales. Esto lleva los argumentos a tres consideraciones básicas. Que el ser humano puede ser pensado ontológicamente como un ser-amor. Que la expresión moral de la vida espiritual es el amor. Que la ES pasa por enseñar a amar, lo que no contradice las intuiciones de Carr y Alexander sobre las virtudes cardinales, aunque las supera integrándolas en orden superior, pues, como dice san Agustín: "La templanza es el amor que totalmente se entrega al objeto amado; la fortaleza es el amor que todo lo soporta por el objeto de sus amores; la justicia es el amor únicamente esclavo de su amado y que ejerce, por lo tanto, señorío conforme a razón; y, finalmente, la prudencia es el amor que con sagacidad y sabiduría elige los medios de defensa contra toda clase de obstáculos" (San Agustín, 1975, pp. 254 y ss.).

En definitiva, parece concluirse que la unidad, la relación y el amor son categorías cruciales por las que discurre una interpretación religiosa de la educación intelectual y la educación moral en el marco de una ES. Pero ¿qué 
sucede cuando esta relación sale del ámbito de lo religioso e intenta tener presencia en el ámbito secular?

\subsection{La discusión sobre la educación del espíritu en el ámbito secular. Lo moral y lo político-legislativo}

Desde una perspectiva secular, la presencia de la ES no se puede delimitar tan claramente en una interpretación intelectiva y otra moral, sino que exige integrarla en la intersección entre lo moral y lo político-legislativo.

La ES desde una perspectiva moral en el ámbito secular no ha sido bien comprendida porque es difícil discernir una moral que pueda brotar de una ES desvinculada de una matriz religiosa. Hand parecía sugerir unas disposiciones espirituales que podían ser educables (Hand, 2003, p. 399), mientras que Jones y De Souza sugerían la compasión (Jones, 2005; De Souza, 2006), del mismo modo que Roebben hablaba de la mutua hospitalidad (Roebben, 2014, pp. 307-309). Pero los argumentos de ambos pueden subsumirse como pequeños matices en un tipo de educación que pensara las consecuencias didácticas de educar el "amor" desde un punto de vista político. Aquí aparece la reciente aportación del profesor Fantuzzo, que sostiene que la vida cívica debe nutrirse del amor que refuerza la solidaridad entre los ciudadanos, pero un amor que nutre no el discurso de los derechos y deberes, sino las relaciones interpersonales de los ciudadanos de a pie (Fantuzzo, 2018, pp. 388-391 y 397-401). Con todo, la discusión sobre el lugar de la ES desde un punto de vista político-legislativo ha dado pie a discusiones de construcción democrática.

Aquí las ideas difractan en dos direcciones. La primera tiene que ver con las propuestas de John Dewey. Pequeñas comunidades compuestas por diversas personas que se ven obligadas a comunicarse para buscar solución a los problemas que les plantea el tiempo histórico que viven: eso es una educación del espíritu desde una perspectiva democrática (Roebben, 2014; Webster, 2009; Hunt, 1953). O, tal y como sugiere Roebben a Dewey, "Learning is in itself a communal enterprise. This process has a spiritual nature, according to Dewey, and could be considered as immanent mysticism" (2014, 
p. 303). Como a medio camino entre los postulados de Dewey y de MacIntyre, Alexander defendía que las learning communities reposaban en la idea de que cada religión tiene una tradición intelectual y moral que transmite a los educandos una interpretación de la existencia que forma el pensamiento crítico y orienta la acción moral; su papel en la construcción de una democracia sería ya no solo que esas tradiciones conviven con los valores cívicos, sino que la propia esencia de la democracia liberal es permitir esas comunidades de aprendizaje que son sostenidas por una ES y facilitar interpretaciones así en la vida pública (Alexander, 2001). Pero falta una consideración por hacer desde el punto de vista de la ES en su relación con la construcción de la democracia. Que si la triada relación-amor-unidad preside la génesis y el desarrollo del espíritu, entonces una educación política desde el punto de vista de una ES debe pasar inexorablemente por esas mismas categorías. Hay algo de esto en las lecturas teológicas de Freire. Y, como Neumann puso de manifiesto, no es solo que la pedagogía crítica "have religious roots and strong connections to liberation theology, but is in and of itself a practice of faith" $(2011,603)$. Acaso Dewey puso esto de manifiesto mejor que el propio Freire cuando escribió su credo pedagógico. Pero ¿a dónde conducen estas observaciones? A dos ideas básicas. Que una educación política basada en una ES se expresa como una estructura de fe que cree en un mundo mejor. Y que ese mundo mejor se construye en las pequeñas acciones de cada día, presididas por el amor, y que tienen como destino a las personas que nos rodean y a la naturaleza, especialmente a las personas más desfavorecidas (Neumann, 2011, pp. 608-609).

En definitiva, no parece que puede pensarse una ES en el ámbito secular desligada completamente de una matriz religiosa. Porque Hand habla de una forma superflua -inusual en él-. Porque Dewey atribuye a la democracia una categoría espiritual. Porque Alexander entiende que la libertad educativa propia de una democracia liberal legitima de por sí una ES. Porque Carr ignora que, incluso dentro de la teología, no hay una lectura unánime de las Escrituras -ni siquiera espiritual-.

$$
* * *
$$

A la sazón, como ya he sugerido, me parece que la manera en que la ES puede pensarse en términos seculares y morales tiene dos grandes dimensio- 
nes que se condensan en dos categorías. Por una parte, la idea de una "unidad relacional" leída en términos gnoseológicos y, por otra parte, la idea de una educación del "amor". Pero estas intuiciones son ya más propias de aparecer en las últimas palabras del artículo.

\section{5. Últimas PALABRAS}

Después de todo el discurso, pareciera que la dificultad de hablar de la ES no procede solo de que no acertemos a describir de forma concisa qué es el espíritu, sino, más todavía, que no conocemos cómo interactúa con el resto de potencias del ser humano. Sin embargo, sí parece posible concluir con una idea básica. Que la ES es la educación de una sensibilidad, de una mirada renovada de la realidad que tiene consecuencias intelectuales, morales, políticas y religiosas.

Esta idea nace de dos presupuestos básicos que han atravesado todos los apartados vistos hasta ahora. Que desde su génesis en la infancia el desarrollo del espíritu reposa en la apertura a todo lo otro sentido o visto o interpretado como una unidad relacional recíproca. Y que la ES infusiona todos los ámbitos educativos donde se considera su educación. De manera que atender a la ES supone, en realidad, atender y resignificar la educación intelectual, moral, religiosa y política. En ese sentido, la Es no se concreta en un ámbito determinado del currículum, mientras que sí se forma en una comunidad. Y es sobre estas líneas que se pueden decir unas últimas palabras.

La relación que mantiene la ES con el intelecto no es la formación de una mente erudita, sino que, sobre ella, se busca una inteligencia capaz de captar el entramado relacional de la realidad, lo que se refleja epistemológicamente en el currículum, y lo subsuma en un orden de sentido superior que guarda relación con la trascendencia. Esto, que nace de la operación del intelecto sobre la realidad, conduce a la dimensión moral. La relación que mantiene la Es con lo moral no acontece en el mero cumplimiento de normas, sino en la "relación" y el "amor" de una forma personal en comunidad. Conceder entidad ontológica a la relación desde un punto de vista moral conduce a una antropología en que el ser humano aparece no solo en relación con otras personas y con la naturaleza, sino en relación de amor. Es decir, en una intuición 
de desear el bien del otro incluso por encima del de uno mismo. O, dicho de otra manera, el ser humano es liberado para amar. Estas mismas intuiciones encuentran acomodo en el pensamiento sobre la sociedad. La relación que mantiene la ES con la educación política va más allá del hecho de que la propia sociedad es un entramado relacional o que existen intuiciones filosóficas que permiten pensar el "amor" en categorías políticas. Es que el lugar de formación propio del espíritu se despliega en una vida comunitaria que no puede reducirse a pequeñas comunidades justificadas por la formación en una tradición compartida. La idea de democracia no se agota en que esto pueda existir sin más, ni puede reducirse a una neutralidad. Debe promover el encuentro de esas comunidades o interpretaciones de la existencia entre sí, es decir, buscar los cauces en que las escuelas se relacionen con otras escuelas o habilitar tantos espacios de formación en una misma escuela como formas de interpretar la realidad existan. Esto se ha desarrollado sobre todo en la promoción de perspectivas ecuménicas y nos lleva a un discurso religioso. La relación que mantiene la ES con la religión es la relación que mantiene el cuerpo y la sangre. Entender la realidad como una unidad relacional revitalizada por el amor a las personas que están cerca no contradice ninguna de las religiones o los carismas propios de cada religión, sino que los infusiona desde dentro y los reconduce a una nueva vida. Así, la oración, la liturgia y la vida sacramentales comprendidas como una relación amorosa con lo divino. En fin, la ES es educar esa sensibilidad.

\section{BiBLIOGRAFÍA}

Adams, K., Bull, R. y Maynes, M.-L. (2015). Early childhood spirituality in education: Towards an understanding of the distinctive features of young children's spirituality. European Early Childhood Education Research Journal 24(5), 760-774. DOI: <https://doi.org/10.1080/135029 3x.2014.996425>.

AleXander, H. A. (2001). Reclaiming goodness. Education and the spiritual quest. Notre Dame, Indiana: University of Notre Dame Press.

Alexander, H. A. (2002). God as teacher: jewish reflection on a Theology of Pedagogy. The Journal of Beliefs and Values 22(1), 5-17. 
AleXander, H. A. (2003). Moral education and liberal democracy: spirituality, community, and character in an open society. Educational Theory, 53(4), 367-387. DOI: $<$ https://doi.org/10.1111/j.1741-5446.2003.00367.x >.

Beringer, A. (2000). In Search of the Sacred: A Conceptual Analysis of Spirituality. Journal of Experiential Education 23(3), 157-165. DOI: <https:// doi.org/10.1177/105382590002300307>.

Benedicto XVI (2007). Sacramentum caritatis. Madrid: San Pablo.

Benedicto XVI (2009). Caritas in Veritate. Madrid: San Pablo.

Ben-Peretz, M. (1975). The concept of curriculum potential. Curriculum Theory Network 5(2), 151-159.

Benson, P. L., Roehlkepartain, E. C. y Rude, S. P. (2003). Spiritual Development in Childhood and Adolescence: Toward a Field of Inquiry. Applied Developmental Science 7(3), 205-213. DOI: <https://doi.org/10.1207/ s1532480xads0703_12>.

Benson, P. L., Scales, P. C., Syvertsen, A. K. y Roehlkepartain, E. C. (2012). Is youth spiritual development a universal developmental process? An international exploration. The Journal of Positive Psychology 7(6), 453-470. DOI: $<$ https://doi.org/10.1080/17439760.2012.732102>.

BEST, R. (2008). In defence of the concept of "spiritual education": a reply to Roger Marples. International Journal of Children's Spirituality 13(4), 321-329. DOI: < https://doi.org/10.1080/13644360802439466>.

Burbules, N. C. (2004). Jesus as Teacher, en H. A. Alexander (ed.), Spirituality and Ethics in Education (pp. 7-20). Brighton: Sussex Academic Press.

Caranfa, A. (2004). Silence as the Foundation of Learning. Educational Theory 54(2), 211-230. DOI: <https://doi.org/10.1111/j.1741-5446.2004.00015.x>.

CARr, D. (1995). Towards a Distinctive Conception of Spiritual Education. Oxford Review of Education 21(1), 83-98. DOI: <https://doi. org/10.1080/0305498950210106>.

CARr, D. (1996). Songs of Immanence and Transcendence: A Rejoinder to Blake. Oxford Review of Education 22(4), 457-463.

CARR, D. (2000). Three conceptions of spirituality for spiritual education, en A. Wright. (ed.), Spirituality and education (pp. 209-221). Londres/Nueva York: Routledge/Falmer.

Cohen, H. (2004). El prójimo. Barcelona: Anthopos. 
Crossman, J. (2003). Secular Spiritual Development in Education from International and Global Perspectives. Oxford Review of Education 29(4), 503-520. DOI: <https://doi.org/10.1080/0305498032000153052>.

Dayton, E. (2015). On the Spiritual Dimension of Education: Finding a Common Ground. Journal of Philosophy of Education 50(3), 432-447. DOI: $<$ https://doi.org/10.1111/1467-9752.12149>.

DeMarzio, D. M. (2017). Rechilding in Educational Theory and Practice: A Patristic Genealogy. Educational Theory 67(3), 265-272. DOI: <https:// doi.org/10.1111/edth.12247>.

De SouzA, M. (2006). Educating for hope, compassion and meaning in a divisive and intolerant world. International Journal of Children's Spirituality 11(1), 165-175. DOI: <https://doi.org/10.1080/13644360500504488>.

De Souza, M. (2016). The spiritual dimension of education. Addressing issues of identity and belonging. Discourse and Communication for Sustainable Education 78(1), 125-138. DOI: <https://doi.org/10.1515/dese-20160009>.

De Souza, M. (2017). The complex reasons for missing spirituality. Democracy \& Education 25(1), 1-7.

Duff, L. (2003). Spiritual Development and Education: a contemplative view. International Journal of Children's Spirituality 8(3), 227-237. DOI: $<$ https://doi.org/10.1080/1364436032000146502>.

Fantuzzo, J. P. (2018). Facing the Civic Love Gap: James Baldwin's Civic Education for interpersonal Solidarity. Educational Theory 68(4-5), 385402. DOI: <https://doi.org/10.1111/edth.12323>.

Filipsone, A. (2009). Religious literacy or spiritual awareness? Comparative critique of Andrew Wright's and David Hay's approaches to spiritual education. International Journal of Children's Spirituality 14(2), 121-128. DOI: <https://doi.org/10.1080/13644360902830242>.

Francisco (2013). Evangelii Gaudium. Madrid: San Pablo.

Francisco (2015). Laudato si'. Madrid: San Pablo.

Francisco (2016). Amoris Laetitia. Madrid: San Pablo.

Francisco (2019). Christus vivit. Madrid: San Pablo.

GARY, K. (2006). Spirituality, critical thinking, and the desire for what is infinite. Studies in Philosophy and Education 25(4), 315-326. DOI: <https:// doi.org/10.1007/s11217-006-9008-0>. 
Geiger, M. W. (2015). Religious Education Person to Person: Attending to Relationality. Religious Education 110(2), 162-180. DOI: <https://doi.org /10.1080/00344087.2015.1013906>.

Goldshmidt, G. (2017). Waldorf Education as Spiritual Education. Religion \& Education 44(3), 346-363. DOI: <https://doi.org/10.1080/15507394.20 $17.1294400>$.

Hand, M. (2003). The Meaning of 'Spiritual Education'. Oxford Review of Education 29(3), 391-401. DOI: <https://doi.org/10.1080/03054980307446>.

Hansen, D. T. (2019). Sor Juana Inés de la Cruz and the Demands of Education. Educational Theory 68(4-5), 443-475.

HaY, D. (2000). Spirituality versus Individualism: Why we should nurture relational consciousness. International Journal of Children's Spirituality 5(1), 37-48. DOI: <https://doi.org/10.1080/713670892>.

Hemmerle, K. (2005). Tras las huellas de Dios. Ontología trinitaria y unidad relacional. Salamanca: Sígueme.

Ho Wong, P. (2006). A conceptual investigation into the possibility of spiritual education. International Journal of Children's Spirituality 11(1), 7385. DOI: <https://doi.org/10.1080/13644360500503407>.

Hunt, T. C. (1953). Moral and spiritual values in education. Educational Theory, 3(4), 374-380. DOI: <https://doi.org/10.1111/j.1741-5446.1953. tb01074.x>.

JoNES, L. (2005). What Does Spirituality in Education Mean? Journal of College and Character 6(7). DOI: <https://doi.org/10.2202/1940-1639.1485>.

Juan Pablo II (1986). El misterio trinitario. Ecclesia de Trinitate, Dives in Misericordia, Redemptor Hominis \& Dominumet Vivificantem. Madrid: BAC.

KiLcup, C. (2016). Secret wisdom. Gifted Education International 32(3), 242257. DOI: <https://doi.org/10.1177/0261429415602587>.

King, P. E. y Boyatzin, Ch. J. (2004). Exploring adolescent spiritual and religious development: current and future theoretical and empirical perspectives. Applied Developmental Science 8(1), 2-6. DOI: <https://doi. org/10.1207/S1532480XADS08010>.

KIRYLO, J. D. (2017). Hate won, but love will have the final word: Critical pedagogy, liberation theology, and the moral imperative of resistance. 
Policy Futures in Education 15(5), 590-601. DOI: <https://doi. org/10.1177/1478210317707454>.

LewIS, J. (2000). Spiritual Education as the Cultivation of Qualities of the Heart and Mind. A Reply to Blake and Carr. Oxford Review of Education 26(2), 263-283. DOI: <https://doi.org/10.1080/713688528>.

Lingley, A. (2016). Democratic foundation for Spiritually responsive pedagogy. Democracy \& Education 24(2), 1-12.

Lovelock, P. y AdAms, K. (2017). From darkness to light: children speak of divine encounter. International Journal of Children's Spirituality 22(1), 36-48. DOI: <https://doi.org/10.1080/1364436x.2016.1268098>.

Marina Díaz, L. (2016). Spiritual Conversation as Religiously Educative. Religious Education 112(5), 477-490. DOI: <https://doi.org/10.1080/003440 87.2015.1113039>.

MARples, R. (2006). Against (the use of the term) "spiritual education." International Journal of Children's Spirituality 11(2), 293-306. DOI: <https:// doi.org/10.1080/13644360600797313>.

Mata-McMaHon, J. (2016). Reviewing the research in children's spirituality (2005-2015): proposing a pluricultural approach. International Journal of Children's Spirituality, 21(2), 140-152. DOI: <https://doi.org/10.1080/136 4436x.2016.1186611>.

Mayes, C. (2002). The teacher as an archetype of spirit. Journal of Curriculum Studies 34(6), 699-718. DOI: <https://doi.org/10.1080 /00220270110118816>.

McCann, J. (2009). An Analysis of the Perspectives of World Religions, en M. de Souza et al. (eds.), International handbook of the religious, moral and spiritual dimensions in education (pp. 917-935). Heidelberg/Londres/ Nueva York: Springer.

Muratore, U. (1998). Antonio Rosmini. Madrid: BAC.

Neumann, J. W. (2011). Critical pedagogy and faith. Educational Theory 61(5), 601-619. DOI: <https://doi.org/10.1111/j.1741-5446.2011.00423.x>.

Newman, J. H. (2009). The Idea of a University. Dublín: International Center for Newman Studies.

RADFORD, M. (2007). Passion and intelligibility in spiritual education. British Journal of Educational Studies 55(1), 21-36. DOI: <https://doi.org/10.111 $1 / \mathrm{j} .1467-8527.2007 .00364 . \mathrm{x}>$. 
ReImer, K. S. y Furrow, J. L. (2001). A Qualitative Exploration of Relational Consciousness in Christian Children. International Journal of Children's Spirituality 6(1), 7-23. DOI: <https://doi.org/10.1080/13644360124074>.

Roebben, B. (2017). Generating Hope: The Future of the Teaching Profession in a Globalized World. Religious Education 112(3), 199-206. DOI: $<$ https://doi.org/10.1080/00344087.2017.1308181>.

Rosmini, A. (2011). Rosmini. Scrittisull'educazione. Brescia: La Scuola.

Ruíz Salvador, F. (1998). Caminos del espíritu. Compendio de teología espiritual. Madrid: Editorial de Espiritualidad.

San Agustín (1975). De moribus Ecclesiaecatholicae et de moribus Manichaeorum, 1, 15, 25, en Obras Completas (4). Madrid: BAC.

Schinkel, A. (2018). Wonder and Moral Education. Educational Theory 68(1), 31-48. DOI: <https://doi.org/10.1111/edth.12287>.

WATson, J. (2011). Discussion in religious education: developing dialogic for community cohesion and/or spiritual development. International Journal of Children's Spirituality 16(2), 97-108. DOI: <https://doi.org/10.1080 /1364436x.2011.580728>.

WeBster, R. S. (2009). The educative value of Dewey's religious attitude for spirituality. International Journal of Children's Spirituality 14(2), 93-103. DOI: <https://doi.org/10.1080/13644360902830168>.

Webster, R. S. (2009). The educative value of Dewey's religious attitude for spirituality. International Journal of Children's Spirituality 14(2), 93-103. DOI: <https://doi.org/10.1080/13644360902830168>.

WILlS, R. (2012). Beyond relation: a critical exploration of "relational consciousness" for spiritual education. International Journal of Children's Spirituality 17(1), 51-60. DOI: <https://doi.org/10.1080 /1364436x.2012.660747>.

Wright, A. (2000). Spirituality and education. Londres/Nueva York: Routledge/Falmer.

Zweiback, Y., Kaplan, S. N. y Manzone, J. (2016). An awareness of spirituality from two perspectives. Gifted Education International 32(3), 232-241. DOI: <https://doi.org/10.1177/0261429415602580>. 
